\title{
Clinical features of patients with acute coronary syndrome during the COVID-19 pandemic
}

\author{
Kensuke Matsushita ${ }^{1,2}$ (D) Sebastien Hess ${ }^{1} \cdot$ Benjamin Marchandot ${ }^{1} \cdot$ Chisato Sato $^{1,2} \cdot$ Dinh Phi Truong $^{1,3}$. \\ Ngoc Thanh $\mathrm{Kim}^{1,3} \cdot$ Anne Weiss ${ }^{4} \cdot$ Laurence Jesel $^{1,2} \cdot$ Patrick Ohlmann ${ }^{1}$ - Olivier Morel ${ }^{1,2}$
}

Accepted: 9 November 2020 / Published online: 16 November 2020

(c) Springer Science+Business Media, LLC, part of Springer Nature 2020

\begin{abstract}
Although a reduction in hospital admissions of acute coronary syndromes (ACS) patients has been observed globally during the coronavirus disease 2019 (COVID-19) pandemic, clinical features of those patients have not been fully investigated. The aim of the present analysis is to investigate the incidence, clinical presentation, and outcomes of patients with ACS during the COVID-19 pandemic. We performed a retrospective analysis of consecutive patients who were admitted for ACS at our institution between March 1 and April 20, 2020 and compared with the equivalent period in 2019. Admissions for acute myocardial infarction (AMI) reduced by $39.5 \%$ in 2020 compared with the equivalent period in 2019 . Owing to the emergency medical services (EMS) of our region, all time components of ST-elevated myocardial infarction care were similar during the COVID-19 outbreak as compared with the previous year's dataset. Among the 106 ACS patients in 2020, 7 patients tested positive for COVID-19. Higher incidence of type 2 myocardial infarction ( $29 \%$ vs. $4 \%, \mathrm{p}=0.0497)$ and elevated D-dimer levels $(5650 \mu \mathrm{g} / 1$ [interquartile range (IQR) $1905-13,625 \mu \mathrm{g} / \mathrm{l}] \mathrm{vs} .400 \mu \mathrm{g} / \mathrm{l}[\mathrm{IQR} 270-1050 \mu \mathrm{g} / \mathrm{l}], \mathrm{p}=0.02)$ were observed in COVID-19 patients. In sum, a significant reduction in admission for AMI was observed during the COVID-19 pandemic. COVID-19 patients were characterized by elevated D-dimer levels on admission, reflecting enhanced COVID-19 related thrombogenicity. The prehospital evaluation by EMS may have played an important role for the timely revascularization for STEMI patients.
\end{abstract}

Keywords Coronavirus disease 2019 - Acute coronary syndrome $\cdot$ Percutaneous coronary intervention · Catheterization laboratory

\section{Highlights}

Electronic supplementary material The online version of this article (https://doi.org/10.1007/s11239-020-02340-z) contains supplementary material, which is available to authorized users.

Olivier Morel

olivier.morel@chru-strasbourg.fr

1 Pôle d'Activité Médico-Chirurgicale Cardio-Vasculaire, Nouvel Hôpital Civil, Centre Hospitalier Universitaire, Université de Strasbourg, 1 place de l'Hôpital, 67091 Strasbourg cedex, France

2 UMR1260 INSERM, Nanomédecine Régénérative, Faculté de Pharmacie, Université de Strasbourg, Illkirch, France

3 Vietnam National Heart Institute, Bach Mai Hospital, Hanoi, Vietnam

4 Centre Hospitalier Universitaire, SAMU 67, Strasbourg, France
- The COVID-19 outbreak was associated with a $40 \%$ decrease in AMI.

- The delay of STEMI care was minimal in our center, suggesting that the healthcare system effectiveness was maintained despite the pandemic.

- ACS patients tested positive for COVID-19 was clearly characterized by elevated D-dimer levels on admission, reflecting enhanced COVID-19 related thrombogenicity.

\section{Introduction}

In December 2019, coronavirus disease 2019 (COVID-19) infected pneumonia caused by severe acute respiratory syndrome coronavirus 2 (SARS-CoV-2) occurred in Wuhan, 
China with further worldwide transmission to a pandemic outbreak [1-5]. A large cluster of COVID-19 occurred in northeast France as early as mid-February, and a substantial number of critically ill patients started to be transferred to our institution (Nouvel Hôpital Civil, Strasbourg, France) from the beginning of March. As a way to contain the disease, the government established stringent lockdown measures as of March 17, 2020. Although a drastic reduction in patient admissions for acute coronary syndrome (ACS) during the confinement was noticed by heath workers $[6$, 7], clinical features of those patients have not been fully investigated [8]. In the present study, we aimed to evaluate incidence, clinical presentation, serial laboratory tests, and clinical outcomes of patients with ACS during the COVID19 pandemic.

\section{Methods}

We conducted a single-center, observational survey to collect data of all consecutive patients who underwent emergency angiography for suspected ACS between March 1 and April 20, 2020 and compared with the equivalent period in 2019. ACS was defined as ST-segment elevation myocardial infarction (STEMI), non-ST-segment elevation myocardial infarction (NSTEMI), or unstable angina pectoris. Symptom-onset-to-first medical contact (FMC) time was defined as the time from patient-reported chest discomfort onset time to the time of FMC. FMC-to-device time was defined as the time from FMC to successful wire crossing time during percutaneous coronary intervention. Door-to-device time was defined as the time from hospital arrival to successful wire crossing time. Catheterization laboratory (cath lab) arrivalto-device time was defined as the time from patient arrival in the cath lab to successful wire crossing time. Based on the World Health Organization guidance, a "confirmed case" of COVID-19 was defined by a positive test of a reversetranscriptase-polymerase-chain-reaction assay of a specimen collected on a nasopharyngeal swab. The COVID-19 testing was performed based on the treating team's discretion. The investigation conforms with the principles outlined in the Declaration of Helsinki.

\section{Statistical analysis}

Categorical variables are expressed as numbers (percentage), and continuous variables are expressed as mean \pm SD or median and inter-quartile values. Differences between 2 groups were assessed with $\chi^{2}$ tests or Fisher's exact tests for categorical variables. Unpaired Student's $t$-test was used to analyze continuous variables with normal distributions, and the Wilcoxon test was used to analyze continuous variables with skewed distributions. Propensity score matching was used to limit the risk of error and the number of biases between the COVID and non-COVID patients. The 3:1 propensity score model was developed using logistic regression. This model included the following covariates: age, sex, STEMI, and NSTEMI. A nearest neighbor algorithm was used to match patients with and without COVID-19 in a $3: 1$ ratio, with a caliper width equal to 0.2 of the standard deviation of the logit of the propensity score. The propensity score matched cohort included a total of 20 patients (5 COVID-19 patients and 15 non-COVID-19 patients). $p$ values of $<0.05$ were considered to indicate statistical significance. All analyses were performed using JMP 13 software ${ }^{\circledR}$ (SAS Institute, Cary, NC) or R version 3.6.3 [9].

\section{Results}

A total of 106 patients were enrolled from the 2020 period and compared with the 174 patients from the equivalent period in 2019 (Table 1). The number of patients with acute myocardial infarction (AMI) was dramatically reduced from 159 in 2019 to 92 in 2020 (39.5\% reduction), whereas the number of patients with STEMI was similar (Fig. 1). Likewise, the number of patient admissions in the cardiology department was reduced by $42 \%$ after the French lockdown (Supplementary Fig. 1).

All time components of STEMI care were similar during the COVID-19 outbreak as compared with the previous year's dataset (Fig. 2). Within the STEMI subgroup, no difference between the 2019 and 2020 cohort could be registered for in-hospital mortality rate ( $4 \%$ vs. $7 \%, \mathrm{p}=1.00)$, median length of hospital stay (4 days [interquartile range (IQR) 3-5 days] vs. 4 days [IQR 3-7 days], $\mathrm{p}=0.81$ ), troponin I levels at baseline $(0.75 \mu \mathrm{g} / \mathrm{l}$ [IQR $0.20-5.64 \mu \mathrm{g} / \mathrm{l}$ ] vs. $1.61 \mu \mathrm{g} / \mathrm{l}$ [IQR $0.16-6.59 \mu \mathrm{g} / \mathrm{l}], \mathrm{p}=0.62)$ and at peak $(88.81 \mu \mathrm{g} / \mathrm{l}$ [IQR $30.07-223.93 \mu \mathrm{g} / \mathrm{l}]$ vs. $81.08 \mu \mathrm{g} / \mathrm{l}$ [IQR $40.68-155.80 \mu \mathrm{g} / \mathrm{l}], \mathrm{p}=0.77)$, baseline brain natriuretic peptide (BNP) levels (36 ng/l [IQR 21-79 ng/l] vs. $65 \mathrm{ng} / \mathrm{l}$ [IQR 31-246 ng/l], $\mathrm{p}=0.17$ ), and left ventricular ejection fraction (LVEF) at baseline $(52 \pm 11 \%$ vs. $50 \pm 12 \%, \mathrm{p}=0.62)$.

Among the 106 ACS patients in 2020, 7 ACS patients tested positive for COVID-19. The clinical features of COVID-19 patients receiving cardiac catheterization for ACS are described in Table 2 and Fig. 3. ACS patients with COVID-19 tended to present with dyspnea (57\% vs. 23\%, $\mathrm{p}=0.07)$ rather than chest pain $(57 \%$ vs. $86 \%, \mathrm{p}=0.08)$. The incidence of STEMI and NSTEMI was similar between patients with and without COVID-19, whereas type 2 myocardial infarction (MI) was more frequent in COVID-19 patients $(29 \%$ vs. $4 \%, p=0.0497)$. Interestingly, not only C-reactive protein levels $(70 \mathrm{mg} / 1$ [IQR $19-267 \mathrm{mg} / \mathrm{l} \mathrm{vs}$. $4 \mathrm{mg} / \mathrm{l}$ [IQR 4-18 mg/l], $\mathrm{p}=0.006$ ) but also D-dimer levels at baseline $(5650 \mu \mathrm{g} / \mathrm{l}$ [IQR 1905-13,625 $\mu \mathrm{g} / \mathrm{l}]$ vs. $400 \mu \mathrm{g} / \mathrm{l}$ 
Table 1 Comparison of patients between 2019 and 2020. $(\mathrm{n}=280)$

\begin{tabular}{|c|c|c|c|}
\hline Characteristic & $\begin{array}{l}2019 \\
(n=174)\end{array}$ & $\begin{array}{l}2020 \\
(n=106)\end{array}$ & $\mathrm{p}$ value \\
\hline COVID-19 positive & $0(0)$ & $7(7)$ & 0.001 \\
\hline Age (years) & $70 \pm 15$ & $67 \pm 13$ & 0.06 \\
\hline Male sex & $114(66)$ & $70(66)$ & 0.93 \\
\hline BMI $\left(\mathrm{kg} / \mathrm{m}^{2}\right)$ & $26.8 \pm 5.1$ & $28.0 \pm 5.7$ & 0.08 \\
\hline \multicolumn{4}{|l|}{ Cardiovascular risk factors } \\
\hline Hypertension & $119(68)$ & $65(61)$ & 0.23 \\
\hline Diabetes mellitus & $55(32)$ & $40(38)$ & 0.29 \\
\hline Dyslipidemia & $94(54)$ & $53(50)$ & 0.51 \\
\hline Current smoker & $39(22)$ & $24(23)$ & 0.96 \\
\hline Chronic kidney disease & $51(29)$ & $18(17)$ & 0.02 \\
\hline Hemodialysis & $0(0)$ & $6(6)$ & 0.002 \\
\hline \multicolumn{4}{|l|}{ Coexisting disorder } \\
\hline Previous MI & $51(29)$ & $23(22)$ & 0.16 \\
\hline Previous PCI & $54(31)$ & $36(34)$ & 0.61 \\
\hline Heart failure & $17(10)$ & $8(8)$ & 0.53 \\
\hline $\mathrm{AF}$ & $29(17)$ & $16(15)$ & 0.73 \\
\hline Stroke & $19(11)$ & $8(8)$ & 0.35 \\
\hline Peripheral artery disease & $33(19)$ & $20(19)$ & 0.98 \\
\hline COPD & $22(13)$ & $12(11)$ & 0.74 \\
\hline History of cancer & $38(22)$ & $5(5)$ & $<0.001$ \\
\hline \multicolumn{4}{|l|}{ Symptoms } \\
\hline Chest pain & $130 / 172(76)$ & $88 / 105(84)$ & 0.10 \\
\hline Dyspnea & $57 / 172(33)$ & $27 / 105(26)$ & 0.19 \\
\hline Fever & $14 / 172(8)$ & $2 / 105(2)$ & 0.03 \\
\hline Cough & $20 / 172(12)$ & $7 / 105(7)$ & 0.18 \\
\hline \multicolumn{4}{|l|}{ Vital signs } \\
\hline Body temperature $\left({ }^{\circ} \mathrm{C}\right)$ & $36.9 \pm 0.8$ & $36.7 \pm 0.6$ & 0.05 \\
\hline Systolic pressure $(\mathrm{mmHg})$ & $137 \pm 27$ & $139 \pm 30$ & 0.68 \\
\hline Diastolic pressure (mmHg) & $75 \pm 15$ & $75 \pm 17$ & 0.97 \\
\hline Pulse rate $(\mathrm{bpm})$ & $84 \pm 22$ & $80 \pm 19$ & 0.14 \\
\hline $\mathrm{SpO}_{2}(\%)$ & $97 \pm 3$ & $96 \pm 3$ & 0.24 \\
\hline Oxygen therapy & 27/173 (16) & $34 / 104(33)$ & $<0.001$ \\
\hline \multicolumn{4}{|l|}{ Diagnosis } \\
\hline STEMI & $40(23)$ & $39(37)$ & 0.01 \\
\hline NSTEMI & $119(68)$ & $53(50)$ & 0.002 \\
\hline Type-1 MI & $117(67)$ & $82(77)$ & 0.07 \\
\hline Type-2 MI & $46(26)$ & $6(6)$ & $<0.001$ \\
\hline Takotsubo cardiomyopathy & $14(8)$ & $4(4)$ & 0.16 \\
\hline Myocarditis & $5(3)$ & $1(1)$ & 0.41 \\
\hline \multicolumn{4}{|l|}{ Echocardiography on admission } \\
\hline LVEF & $50 \pm 14$ & $49 \pm 14$ & 0.71 \\
\hline \multicolumn{4}{|l|}{ Lesion characteristics } \\
\hline Single-vessel disease & $42(24)$ & $45(42)$ & 0.001 \\
\hline Multi-vessel disease & $84(48)$ & $47(44)$ & 0.43 \\
\hline \multicolumn{4}{|l|}{ Types of intervention } \\
\hline PCI & $105(60)$ & $79(75)$ & 0.02 \\
\hline POBA & $0(0)$ & $1(1)$ & 0.38 \\
\hline Thrombolysis & $0(0)$ & $0(0)$ & - \\
\hline CABG & $4(2)$ & $0(0)$ & 0.30 \\
\hline Blood examination on admiss & & & \\
\hline
\end{tabular}


Table 1 (continued)

\begin{tabular}{|c|c|c|c|}
\hline Characteristic & $\begin{array}{l}2019 \\
(n=174)\end{array}$ & $\begin{array}{l}2020 \\
(n=106)\end{array}$ & $\mathrm{p}$ value \\
\hline $\operatorname{WBC}\left(10^{9} / 1\right)$ & $10.9 \pm 4.8$ & $10.2 \pm 3.9$ & 0.20 \\
\hline $\mathrm{Hb}(\mathrm{g} / \mathrm{dl})$ & $12.8 \pm 2.4$ & $13.3 \pm 2.3$ & 0.12 \\
\hline Platelets $\left(10^{9} / 1\right)$ & $251 \pm 89$ & $242 \pm 83$ & 0.38 \\
\hline Troponin I $(\mu \mathrm{g} / \mathrm{l})$ & $0.64(0.14-3.44)$ & $0.38(0.08-2.93)$ & 0.17 \\
\hline $\mathrm{Cr}(\mu \mathrm{mol} / \mathrm{l})$ & $75(62-96)$ & $79(62-95)$ & 0.57 \\
\hline eGFR $\left(\mathrm{ml} / \mathrm{min} / 1.73 \mathrm{~m}^{2}\right)$ & $76 \pm 26$ & $76 \pm 28$ & 0.87 \\
\hline LDL-C (g/l) & $0.95 \pm 0.38$ & $1.04 \pm 0.45$ & 0.11 \\
\hline HDL-C (g/l) & $0.43 \pm 0.15$ & $0.45 \pm 0.17$ & 0.48 \\
\hline HbA1c (\%) & $6.2 \pm 1.3$ & $6.5 \pm 1.4$ & 0.19 \\
\hline $\mathrm{BNP}(\mathrm{ng} / \mathrm{l})$ & $183(45-685)$ & $222(51-480)$ & 0.68 \\
\hline $\mathrm{CRP}(\mathrm{mg} / \mathrm{l})$ & $7(4-28)$ & $4(4-89)$ & 0.17 \\
\hline D-dimer $(\mu \mathrm{g} / \mathrm{l})$ & $840(355-2255)$ & $410(270-1130)$ & 0.003 \\
\hline \multicolumn{4}{|l|}{ In-hospital events } \\
\hline Hospital length of stay (days) & $6(3-12)$ & $4(3-7)$ & $<0.001$ \\
\hline ICU stay (days) & $3(2-5)$ & $2(0-3)$ & $<0.001$ \\
\hline In-hospital death & $12 / 174(7)$ & $7 / 103(7)$ & 0.97 \\
\hline New-onset AF & $24 / 174(14)$ & 8/103 (8) & 0.13 \\
\hline Stroke & $9 / 174(5)$ & $3 / 103(3)$ & 0.54 \\
\hline Venous thromboembolism & $9 / 171(5)$ & $2 / 103(2)$ & 0.22 \\
\hline VT/VF & 29/174 (17) & $2 / 103(2)$ & $<0.001$ \\
\hline ARDS & $0 / 174(0)$ & $2 / 103(2)$ & 0.14 \\
\hline Acute kidney injury & $37 / 174(21)$ & 15/104 (14) & 0.16 \\
\hline Multiple organ failure & $13 / 174(7)$ & $1 / 103(1)$ & 0.02 \\
\hline
\end{tabular}

Values are $\mathrm{n}(\%), \mathrm{n} / \mathrm{N}(\%)$, mean $\pm \mathrm{SD}$, or median (interquartile range)

$A F$ atrial fibrillation, $A R D S$ acute respiratory distress syndrome, $B M I$ body mass index, $B N P$ brain natriuretic peptide, $C A B G$ coronary artery bypass grafting, $C O P D$ chronic obstructive pulmonary disease, COVID-19 coronavirus disease 2019, $C r$ creatinine, CRP C-reactive protein, $e G F R$ estimated glomerular filtration rate, $H b$ hemoglobin, $H D L-C$ high-density lipoprotein cholesterol, $I C U$ intensive care unit, $L D L$ $C$ low-density lipoprotein cholesterol, $L V E F$ left ventricular ejection fraction, $M I$ myocardial infarction, NSTEMI non-ST-segment elevation myocardial infarction, $P C I$ percutaneous coronary intervention, $P O B A$ plain old balloon angioplasty, STEMI ST-segment elevation myocardial infarction, $V F$ ventricular fibrillation, $V T$ ventricular tachycardia, $W B C$ white blood cell

[IQR 270-1050 $\mu \mathrm{g} / \mathrm{l}], \mathrm{p}=0.02$ ) were significantly higher in COVID-19 patients than non-COVID-19 patients. COVID19 patients had longer hospital stay (16 days [IQR 3-18 days vs. 4 days [IQR 2-6 days], $\mathrm{p}=0.03$ ) and higher incidence of acute respiratory distress syndrome ( $29 \%$ vs. $0 \%, \mathrm{p}=0.003)$ and venous thromboembolism $(29 \%$ vs. $0 \%, \mathrm{p}=0.003)$. Further analysis utilizing 3:1 propensity score matching demonstrated similar trends in D-dimer levels, hospital length of stay and acute respiratory distress syndrome (ARDS), which did not reach statistical significance, probably due to the small number of patients included (Supplementary Table 1).

\section{Discussion}

In the present study, the COVID-19 outbreak was associated with a $40 \%$ decrease in AMI. Similar findings have been noted in northern Italy and in the United States [10,
11]. The identification of the mechanisms leading to the reduction in admissions for AMI is beyond the scope of the present work. Nevertheless, recent reports suggested that the decreased number of ACS patients was due to the fear of exposure to COVID-19 affected subjects at hospital admission or a true reduction in the incidence of ACS as the potential result of low physical stress during the social containment $[6,12,13]$.

The delay on STEMI reperfusion during the COVID-19 pandemic has been explained by the fact that the emergency medical system was focused on COVID-19 and a large number of healthcare workers was relocated to manage the pandemic, resulting in increased mortality and complications in STEMI patients $[6,14,15]$. In contrast, the delay of STEMI care was minimal in our center, suggesting that the healthcare system effectiveness was maintained despite the pandemic. Owing to the emergency medical services (EMS) of our region, more than half of 


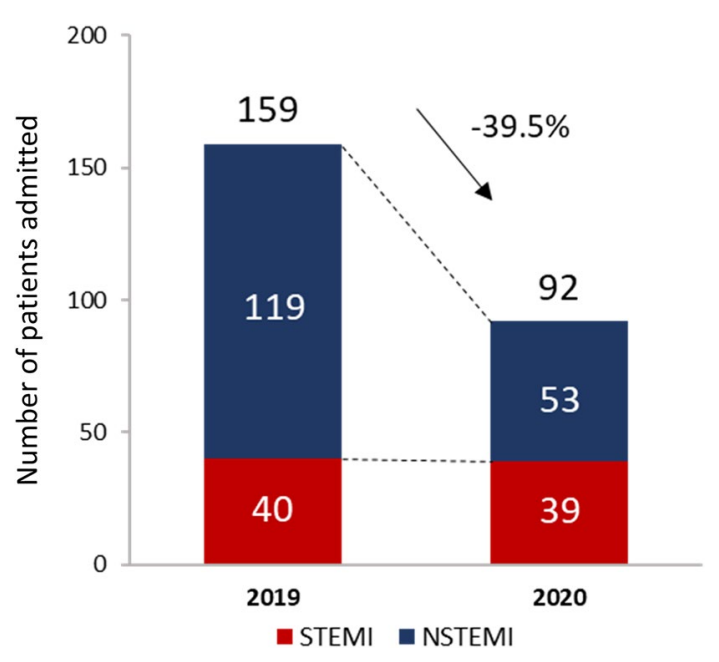

Fig. 1 Numbers of myocardial infarction before and after COVID-19 pandemic. COVID-19 coronavirus disease 2019, NSTEMI non-STsegment elevation myocardial infarction, STEMI ST-segment elevation myocardial infarction

the STEMI patients continued to be directly referred to the cath lab according to prehospital electrocardiograms (60\% vs. $73 \%, p=0.29$ ).

In the present study, patients tested positive for COVID-19 tended to have less chest pain and more type 2 MI. Prior investigations suggested that those patients may have hypoxemia or hypotension along with intense systemic inflammation, which may cause an oxygen supply and demand imbalance in the heart, especially when underlying CAD exists [16-18]. Moreover, COVID-19 patients were clearly characterized by elevated D-dimer levels on admission in the present study, reflecting enhanced COVID-19 related thrombogenicity [19-22]. Although the increased thrombotic susceptibility in COVID-19 patients are far beyond the scope of this study, prior studies underlined that higher thrombotic burden in the acute phase of COVID-19 relies on pro-inflammatory cytokine/chemokine release [23], increased endothelial dysfunction/damage, and potential sepsis induced coagulopathy development in severe cases, all promoting coagulation activation. A recent observational study including 115 STEMI patients clarified that COVID-19 patients had an elevated D-dimer levels as well as higher thrombotic burden compared to non-COVID patients [24]. Our study described lower PCI rate and higher incidence of venous thromboembolism and ARDS in the COVID-19 patients (Table 2), suggesting that those complications may have led COVID-19 patients to present ACS like symptoms. These insights may pave the way toward a novel diagnostic perspective for ACS patients complicated to COVID-19.

\section{Study limitation}

We acknowledge the following limitations: First, the analyses were performed on the basis of a single center data set with uncertain generalizability. Second, owing to the retrospective nature of this study, there were inherent limitations related to cofounding known or unknown factors. Third, the study period was limited to 7 weeks based on the pandemic period in our region. The use of such a limited period for data collection may represent a source for potential bias. Forth, the effect of the low event rate might have been more pronounced when analyzing small subgroups. Fifth, the incidence of diagnostic testing was only $27 \%$ in the non-COVID-19 group, suggesting that certain asymptomatic COVID-19 patients may have been mis-classified. Sixth, a large proportion of COVID19 patients complicated by AMI was not due to coronary obstruction but due to increased thrombogenicity and/or inflammation. The true effects of COVID-19 on coronary arteries should be identified by a larger cohort.

\section{Conclusions}

In conclusion, a significant reduction in admission for AMI was observed during the COVID-19 pandemic. The prehospital evaluation by EMS may have played an important role for the timely revascularization for STEMI patients. Further studies are needed for the establishment of a dedicate diagnostic pathway for ACS patients with COVID-19, aimed at minimizing healthcare providers risk of infection. 
Fig. 2 Time components of STEMI care before and after COVID-19 pandemic. COVID 19 coronavirus disease 2019 ,

FMC first medical contact,

STEMI ST-segment elevation myocardial infarction
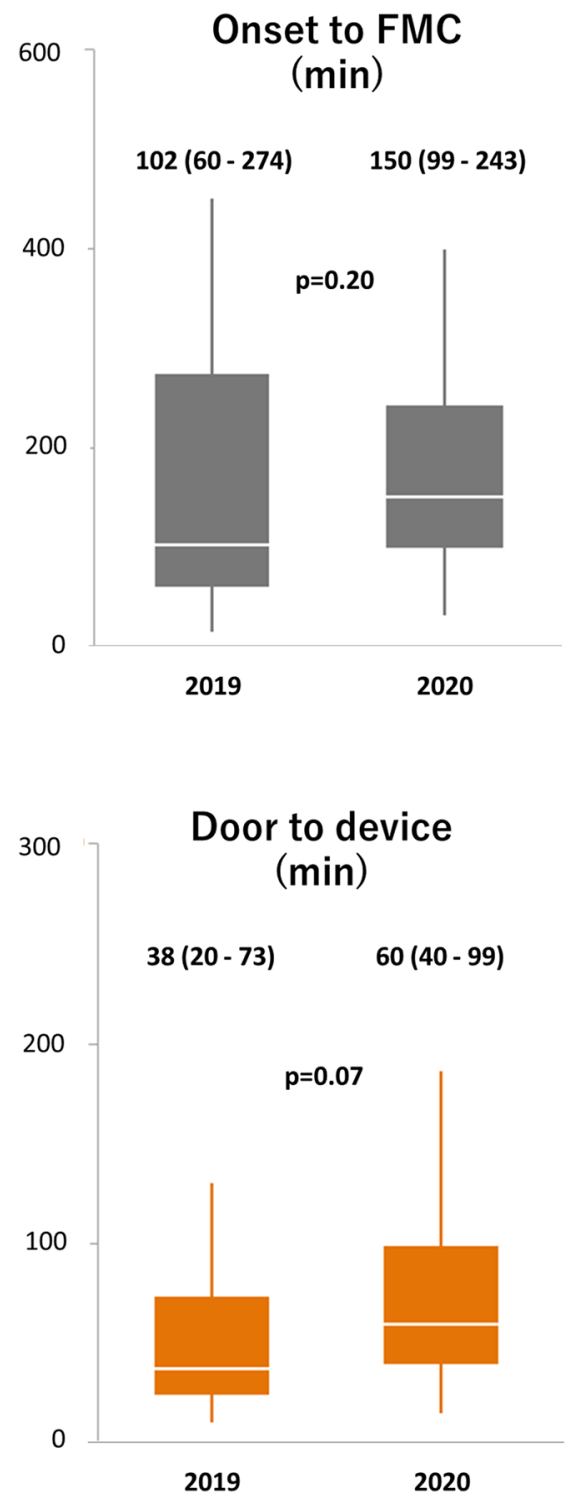

300

FMC to device (min)

$102(82-114) \quad 95(83-123)$

200

100

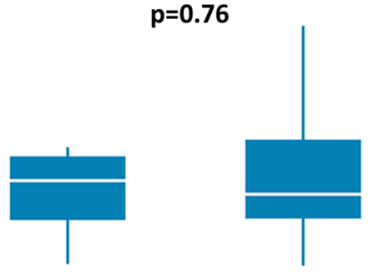

$p=0.76$

2019

2020

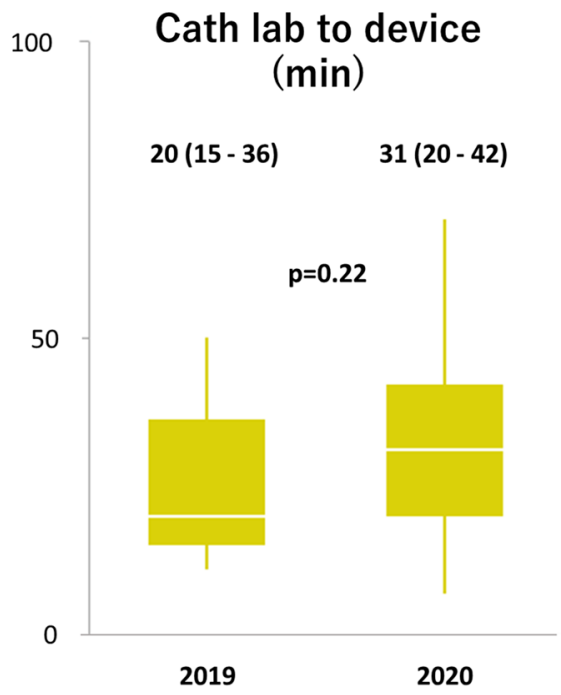


Table 2 Clinical characteristics of patients with and without COVID-19 in 2020. $(n=106)$

\begin{tabular}{|c|c|c|c|}
\hline Characteristic & $\begin{array}{l}\text { COVID-19+ } \\
(n=7)\end{array}$ & $\begin{array}{l}\text { COVID-19- } \\
(\mathrm{n}=99)\end{array}$ & $\mathrm{p}$ value \\
\hline Age (years) & $69 \pm 18$ & $66 \pm 13$ & 0.60 \\
\hline Male sex & $5(71)$ & $65(66)$ & 1.00 \\
\hline BMI $\left(\mathrm{kg} / \mathrm{m}^{2}\right)$ & $28.7 \pm 9.8$ & $28.0 \pm 5.6$ & 0.81 \\
\hline \multicolumn{4}{|l|}{ Cardiovascular risk factors } \\
\hline Hypertension & $3(43)$ & $62(62)$ & 0.43 \\
\hline Diabetes mellitus & $3(43)$ & $37(37)$ & 1.00 \\
\hline Dyslipidemia & $1(14)$ & $52(53)$ & 0.11 \\
\hline Current smoker & $2(29)$ & $22(22)$ & 0.66 \\
\hline Chronic kidney disease & $0(0)$ & $10(18)$ & 0.60 \\
\hline Hemodialysis & $(0)$ & $6(6)$ & 1.00 \\
\hline \multicolumn{4}{|l|}{ Coexisting disorder } \\
\hline Previous MI & $0(0)$ & $23(23)$ & 0.34 \\
\hline Previous PCI & $2(29)$ & $34(34)$ & 1.00 \\
\hline Heart failure & $1(14)$ & $7(7)$ & 0.43 \\
\hline $\mathrm{AF}$ & $1(14)$ & $15(15)$ & 1.00 \\
\hline Stroke & $1(14)$ & $7(7)$ & 0.43 \\
\hline Peripheral artery disease & $1(14)$ & $19(19)$ & 1.00 \\
\hline COPD & $1(14)$ & $11(11)$ & 0.58 \\
\hline History of cancer & $2(29)$ & $3(3)$ & 0.03 \\
\hline \multicolumn{4}{|l|}{ Symptoms } \\
\hline Chest pain & $4(57)$ & $84(86)$ & 0.08 \\
\hline Dyspnea & $4(57)$ & $23(23)$ & 0.07 \\
\hline Fever & $1(14)$ & $1(1)$ & 0.13 \\
\hline Cough & $2(29)$ & $5(5)$ & 0.07 \\
\hline \multicolumn{4}{|l|}{ Vital signs } \\
\hline Body temperature $\left({ }^{\circ} \mathrm{C}\right)$ & $36.9 \pm 1.24$ & $36.7 \pm 0.6$ & 0.41 \\
\hline Systolic pressure $(\mathrm{mmHg})$ & $121 \pm 26$ & $139 \pm 30$ & 0.19 \\
\hline Diastolic pressure (mmHg) & $64 \pm 13$ & $76 \pm 17$ & 0.12 \\
\hline Pulse rate (bpm) & $82 \pm 24$ & $80 \pm 19$ & 0.86 \\
\hline $\mathrm{SpO} 2(\%)$ & $96 \pm 1$ & $96 \pm 3$ & 0.90 \\
\hline Oxygen therapy & $5 / 7(71)$ & 29/97 (30) & 0.04 \\
\hline \multicolumn{4}{|l|}{ Diagnosis } \\
\hline STEMI & $2(29)$ & $37(37)$ & 0.71 \\
\hline NSTEMI & $3(43)$ & $50(51)$ & 1.00 \\
\hline Type-1 MI & $3(43)$ & $79(80)$ & 0.04 \\
\hline Type-2 MI & $2(29)$ & $4(4)$ & 0.0497 \\
\hline Takotsubo cardiomyopathy & $0(0)$ & $4(4)$ & 1.00 \\
\hline Myocarditis & $1(14)$ & $0(0)$ & 0.07 \\
\hline \multicolumn{4}{|l|}{ Echocardiography on admission } \\
\hline $\operatorname{LVEF}(\%)$ & $48 \pm 10$ & $49 \pm 14$ & 0.77 \\
\hline \multicolumn{4}{|c|}{ Blood examination on admission } \\
\hline WBC $\left(10^{9} / 1\right)$ & $8.6 \pm 3.1$ & $10.3 \pm 4.0$ & 0.30 \\
\hline $\mathrm{Hb}(\mathrm{g} / \mathrm{dl})$ & $12.6 \pm 1.4$ & $13.3 \pm 2.3$ & 0.44 \\
\hline Platelets $\left.10^{9} / 1\right)$ & $177 \pm 119$ & $246 \pm 79$ & 0.047 \\
\hline Troponin I ( $\mu \mathrm{g} / \mathrm{l})$ & $0.62(0.01-1.52)$ & $0.38(0.08-3.15)$ & 0.32 \\
\hline $\mathrm{Cr}(\mu \mathrm{mol} / \mathrm{l})$ & $82(62-99)$ & $79(62-95)$ & 0.97 \\
\hline eGFR (ml/min/1.73 m²) & $81 \pm 23$ & $76 \pm 28$ & 0.71 \\
\hline LDL-C (g/l) & $0.93 \pm 0.47$ & $1.04 \pm 0.46$ & 0.68 \\
\hline HDL-C (g/l) & $0.54 \pm 0.27$ & $0.44 \pm 0.17$ & 0.31 \\
\hline HbA1c $(\%)$ & $5.8 \pm 0.4$ & $6.5 \pm 1.5$ & 0.41 \\
\hline
\end{tabular}


Table 2 (continued)

\begin{tabular}{lllc}
\hline Characteristic & $\begin{array}{l}\text { COVID-19+ } \\
(\mathrm{n}=7)\end{array}$ & $\begin{array}{l}\text { COVID-19- } \\
(\mathrm{n}=99)\end{array}$ & p value \\
\hline BNP (ng/l) & $324(138-1068)$ & $197(45-480)$ & 0.33 \\
CRP (mg/l) & $70(19-267)$ & $4(4-18)$ & 0.006 \\
D-dimer $(\mu \mathrm{g} / \mathrm{l})$ & $5650(1905-13,625)$ & $400(270-1050)$ & 0.02 \\
COVID-19 diagnostic testing performed & $7(100)$ & $27(27)$ & $<0.001$ \\
Lesion characteristics & & & \\
Single-vessel disease & $4(57)$ & $41(41)$ & 0.45 \\
Multi-vessel disease & $1(14)$ & $46(46)$ & 0.13 \\
Types of intervention & & & \\
PCI & $3(43)$ & $76(77)$ & 0.07 \\
POBA & $0(0)$ & $1(1)$ & 1.00 \\
Thrombolysis & $0(0)$ & $0(0)$ & - \\
CABG & $0(0)$ & $0(0)$ & - \\
In-hospital events & & $4(2-6)$ & 0.03 \\
Hospital length of stay, days & $16(3-18)$ & $2(0-3)$ & 0.24 \\
ICU stay (days) & $9(0-11)$ & $6 / 97(6)$ & 0.35 \\
In-hospital death & $1 / 6(17)$ & $8 / 97(8)$ & 1.00 \\
New-onset AF & $0 / 6(0)$ & $2 / 97(2)$ & 0.17 \\
Stroke & $1 / 6(17)$ & $0 / 97(0)$ & 0.003 \\
Venous thromboembolism & $2 / 6(33)$ & $2 / 97(2)$ & 1.00 \\
VT/VF & $0 / 6(0)$ & $0 / 97(0)$ & 0.003 \\
ARDS & $2 / 6(33)$ & $13 / 97(13)$ & 0.27 \\
Acute kidney injury & $2 / 6(29)$ & & - \\
Multiple organ failure & $0 / 6(0)$ & & \\
\hline & & & \\
\hline
\end{tabular}

Values are $\mathrm{n}(\%), \mathrm{n} / \mathrm{N}(\%)$, mean $\pm \mathrm{SD}$, or median (interquartile range)

$A F$ atrial fibrillation, $A R D S$ acute respiratory distress syndrome, $B M I$ body mass index, $B N P$ brain natriuretic peptide, $C A B G$ coronary artery bypass grafting, $C O P D$ chronic obstructive pulmonary disease, COVID-19 coronavirus disease 2019, $C r$ creatinine, CRP C-reactive protein, $e G F R$ estimated glomerular filtration rate, $H b$ hemoglobin, $H D L-C$ high-density lipoprotein cholesterol, $I C U$ intensive care unit, $L D L$ $C$ low-density lipoprotein cholesterol, $L V E F$ left ventricular ejection fraction, $M I$ myocardial infarction, NSTEMI non-ST-segment elevation myocardial infarction, $P C I$ percutaneous coronary intervention, $P O B A$, plain old balloon angioplasty, STEMI ST-segment elevation myocardial infarction, $V F$ ventricular fibrillation, $V T$ ventricular tachycardia, $W B C$ white blood cell 


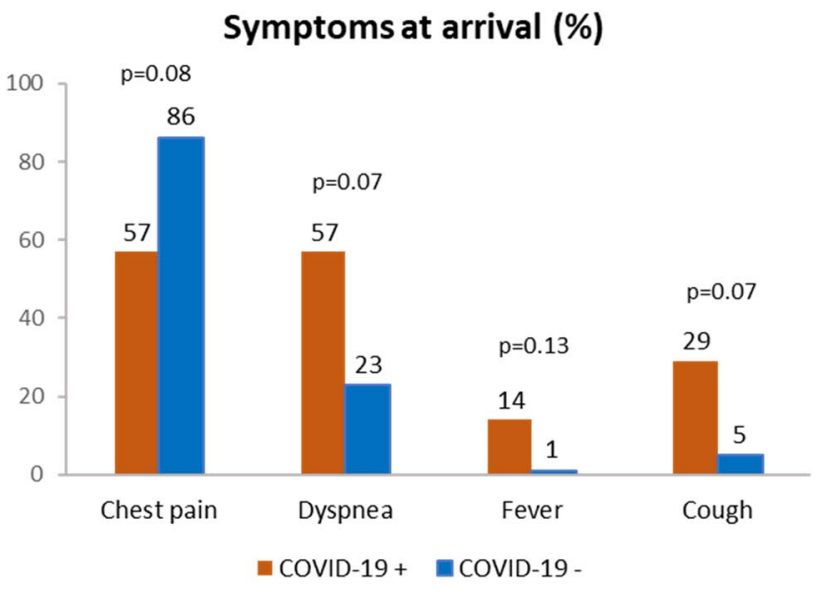

Types of AMI (\%)

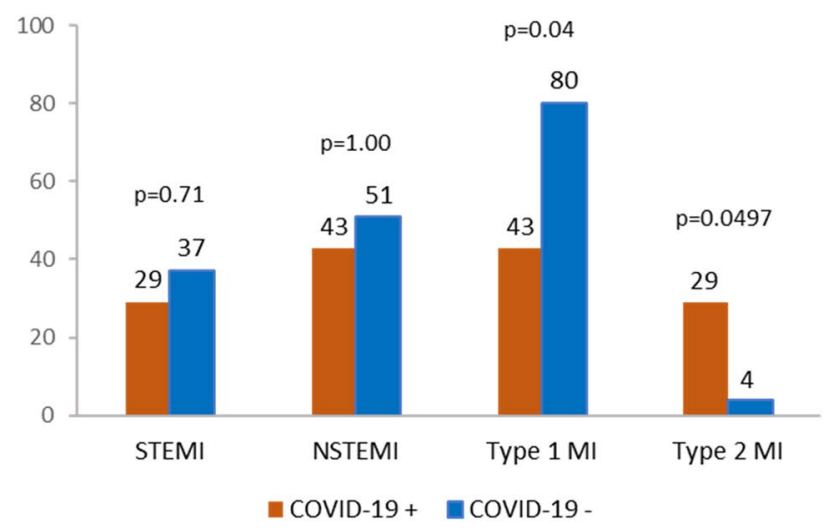

Admission laboratory tests

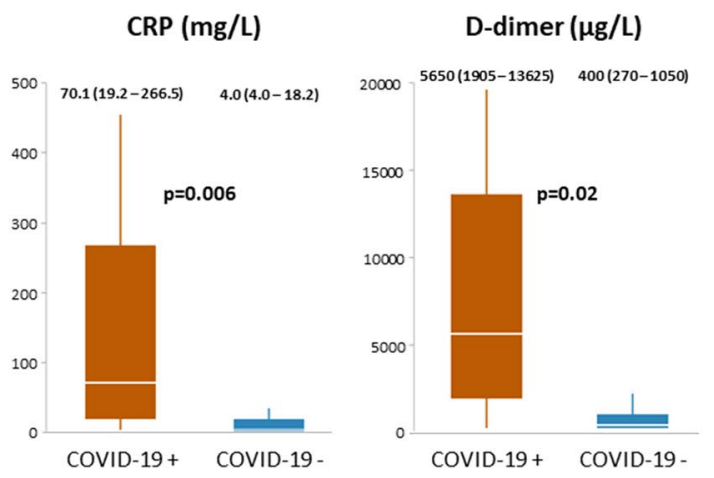

Fig. 3 Clinical features of COVID-19 patients undergoing cardiac catheterization for ACS. ACS acute coronary syndrome, AMI acute myocardial infarction, COVID-19 coronavirus disease 2019, CRP C-reactive protein, $M I$ myocardial infarction, NSTEMI non-ST-segment elevation myocardial infarction, STEMI ST-segment elevation myocardial infarction

Funding This work was supported by GERCA (Groupe pour l'Enseignement, la Recherche Cardiologique en Alsace).

\section{Compliance with ethical standards}

Conflict of interest The authors declare that they have no conflict of interest.

Ethical approval The present study was approved by the Research Ethics Committee of Strasbourg Hospital (CE-2020-69) and the requirement for informed consent was waived by the Ethics Committee.

\section{References}

1. Guan WJ, Ni ZY, Hu Y, Liang WH, Ou CQ, He JX, Liu L, Shan H, Lei CL, Hui DSC, Du B, Li LJ, Zeng G, Yuen KY, Chen RC, Tang CL, Wang T, Chen PY, Xiang J, Li SY, Wang JL, Liang ZJ, Peng YX, Wei L, Liu Y, Hu YH, Peng P, Wang JM, Liu JY, Chen Z, Li G, Zheng ZJ, Qiu SQ, Luo J, Ye CJ, Zhu SY, Zhong NS (2020) Clinical characteristics of coronavirus disease 2019 in China. N Engl J Med. https://doi.org/10.1056/NEJMoa2002032

2. Huang C, Wang Y, Li X, Ren L, Zhao J, Hu Y, Zhang L, Fan G, Xu J, Gu X, Cheng Z, Yu T, Xia J, Wei Y, Wu W, Xie X, Yin W, Li H, Liu M, Xiao Y, Gao H, Guo L, Xie J, Wang G, Jiang R, Gao Z, Jin Q, Wang J, Cao B (2020) Clinical features of patients infected with 2019 novel coronavirus in Wuhan, China. Lancet 1:11. https://doi.org/10.1016/s0140-6736(20)30183-5

3. Matsushita K, Marchandot B, Jesel L, Ohlmann P, Morel O (2020) Impact of COVID-19 on the cardiovascular system: a review. J Clin Med. https://doi.org/10.3390/jcm9051407

4. Sugimoto T, Mizuno A, Kishi T, Ito N, Matsumoto C, Fukuda M, Kagiyama N, Shibata T, Ohmori T, Oishi S, Fuse J, Kida K, Kawai F, Ishida M, Sanada S, Komuro I, Node K (2020) Coronavirus disease 2019 (COVID-19) information for cardiologists - systematic literature review and additional analysis. Circ J. https ://doi.org/10.1253/circj.CJ-20-0302

5. Xie Y, You Q, Wu C, Cao S, Qu G, Yan X, Han X, Wang C, Zhang $\mathrm{H}$ (2020) Impact of cardiovascular disease on clinical characteristics and outcomes of coronavirus disease 2019 (COVID-19). Circ J. https://doi.org/10.1253/circj.CJ-20-0348

6. De Rosa S, Spaccarotella C, Basso C, Calabrò MP, Curcio A, Filardi PP, Mancone M, Mercuro G, Muscoli S, Nodari S, Pedrinelli R, Sinagra G, Indolfi C (2020) Reduction of hospitalizations for myocardial infarction in Italy in the COVID-19 era. Eur Heart J. https://doi.org/10.1093/eurheartj/ehaa409

7. Vlachakis PK, Tentolouris A, Kanakakis I (2020) Concerns for management of STEMI patients in the COVID-19 era: a paradox phenomenon. J Thromb Thrombol. https://doi.org/10.1007/ s11239-020-02236-y

8. Stefanini GG, Montorfano M, Trabattoni D, Andreini D, Ferrante G, Ancona M, Metra M, Curello S, Maffeo D, Pero G, Cacucci M, Assanelli E, Bellini B, Russo F, Ielasi A, Tespili M, Danzi GB, Vandoni P, Bollati M, Barbieri L, Oreglia J, Lettieri C, Cremonesi A, Carugo S, Reimers B, Condorelli G, Chieffo A (2020) ST-elevation myocardial infarction in patients with COVID-19: clinical and angiographic outcomes. Circulation. https://doi.org/10.1161/circulationaha.120.047525

9. Kanda Y (2013) Investigation of the freely available easy-to-use software "EZR" for medical statistics. Bone Marrow Transpl. https://doi.org/10.1038/bmt.2012.244

10. De Filippo O, D’Ascenzo F, Angelini F, Bocchino PP, Conrotto F, Saglietto A, Secco GG, Campo G, Gallone G, Verardi R, Gaido L, Iannaccone M, Galvani M, Ugo F, Barbero U, Infantino V, Olivotti L, Mennuni M, Gili S, Infusino F, Vercellino M, Zucchetti O, Casella G, Giammaria M, Boccuzzi G, Tolomeo P, 
Doronzo B, Senatore G, Grosso Marra W, Rognoni A, Trabattoni D, Franchin L, Borin A, Bruno F, Galluzzo A, Gambino A, Nicolino A, Truffa Giachet A, Sardella G, Fedele F, Monticone S, Montefusco A, Omedè P, Pennone M, Patti G, Mancone M, De Ferrari GM (2020) Reduced rate of hospital admissions for ACS during COVID-19 outbreak in Northern Italy. N Engl J Med. https ://doi.org/10.1056/NEJMc2009166

11. Solomon MD, McNulty EJ, Rana JS, Leong TK, Lee C, Sung S-H, Ambrosy AP, Sidney S, Go AS (2020) The Covid-19 pandemic and the incidence of acute myocardial infarction. N Engl J Med. https://doi.org/10.1056/NEJMc2015630

12. Piccolo R, Bruzzese D, Mauro C, Aloia A, Baldi C, Boccalatte M, Bottiglieri G, Briguori C, Caiazzo G, Calabrò P, CappelliBigazzi M, De Simone C, Di Lorenzo E, Golino P, Monda V, Perrotta R, Quaranta G, Russolillo E, Scherillo M, Tesorio T, Tuccillo B, Valva G, Villari B, Tarantini G, Varricchio A, Esposito G (2020) Population trends in rates of percutaneous coronary revascularization for acute coronary syndromes associated with the COVID-19 outbreak. Circulation. https://doi.org/10.1161/circu lationaha.120.047457

13. Garcia S, Albaghdadi MS, Meraj PM, Schmidt C, Garberich R, Jaffer FA, Dixon S, Rade JJ, Tannenbaum M, Chambers J, Huang PP, Henry TD (2020) Reduction in ST-segment elevation cardiac catheterization laboratory activations in the United States during COVID-19 pandemic. J Am Coll Cardiol. https://doi. org/10.1016/j.jacc.2020.04.011

14. Tam CF, Cheung KS, Lam S, Wong A, Yung A, Sze M, Lam YM, Chan C, Tsang TC, Tsui M, Tse HF, Siu CW (2020) Impact of coronavirus disease 2019 (COVID-19) outbreak on ST-segmentelevation myocardial infarction care in Hong Kong, China. Circ Cardiovasc Qual Outcomes 1:11. https://doi.org/10.1161/circo utcomes. 120.006631

15. Ferlini M, Andreassi A, Carugo S, Cuccia C, Bianchini B, Castiglioni B, Guagliumi G, Lettieri C, Lettino M, Marenzi G, Metra M, Migliori M, Montorfano M, Oliva F, Savonitto S, Seregni R, Visconti LO (2020) Centralization of the ST elevation myocardial infarction care network in the Lombardy region during the COVID-19 outbreak. Int J Cardiol. https://doi.org/10.1016/j.ijcar d.2020.04.062

16. Guo T, Fan Y, Chen M, Wu X, Zhang L, He T, Wang H, Wan J, Wang X, Lu Z (2020) Cardiovascular implications of fatal outcomes of patients with coronavirus disease 2019 (COVID-19). JAMA Cardiol. https://doi.org/10.1001/jamacardio.2020.1017
17. Li B, Yang J, Zhao F, Zhi L, Wang X, Liu L, Bi Z, Zhao Y (2020) Prevalence and impact of cardiovascular metabolic diseases on COVID-19 in China. Clin Res Cardiol. https://doi.org/10.1007/ s00392-020-01626-9

18. Thygesen K, Alpert JS, Jaffe AS, Chaitman BR, Bax JJ, Morrow DA, White HD (2018) Fourth universal definition of myocardial infarction (2018). J Am Coll Cardiol. https://doi.org/10.1016/j. jacc.2018.08.1038

19. Marchandot B, Sattler L, Jesel L, Matsushita K, Schini-Kerth V, Grunebaum L, Morel O (2020) COVID-19 related coagulopathy: a distinct entity? J Clin Med. https://doi.org/10.3390/jcm9061651

20. Faggiano P, Bonelli A, Paris S, Milesi G, Bisegna S, Bernardi N, Curnis A, Agricola E, Maroldi R (2020) Acute pulmonary embolism in COVID-19 disease: preliminary report on seven patients. Int J Cardiol. https://doi.org/10.1016/j.ijcard.2020.04.028

21. Hasan SS, Radford S, Kow CS, Zaidi STR (2020) Venous thromboembolism in critically ill COVID-19 patients receiving prophylactic or therapeutic anticoagulation: a systematic review and meta-analysis. J Thromb Thrombol. https://doi.org/10.1007/s1123 9-020-02235-Z

22. Marchandot B, Trimaille A, Curtiaud A, Matsushita K, Jesel L, Morel O (2020) Thromboprophylaxis: balancing evidence and experience during the COVID-19 pandemic. J Thromb Thrombol. https://doi.org/10.1007/s11239-020-02231-3

23. Lin L, Lu L, Cao W, Li T (2020) Hypothesis for potential pathogenesis of SARS-CoV-2 infection-a review of immune changes in patients with viral pneumonia. Emerg Microbes Infect. https:// doi.org/10.1080/22221751.2020.1746199

24. Choudry FA, Hamshere SM, Rathod KS, Akhtar MM, Archbold RA, Guttmann OP, Woldman S, Jain AK, Knight CJ, Baumbach A, Mathur A, Jones DA (2020) High thrombus burden in patients with COVID-19 presenting with ST-segment elevation myocardial infarction. J Am Coll Cardiol. https://doi.org/10.1016/j. jacc.2020.07.022

Publisher's Note Springer Nature remains neutral with regard to jurisdictional claims in published maps and institutional affiliations. 